\title{
Psychogeriatrics in Nigeria
}

\author{
A. O. Ogunlesi, Senior Consultant Psychiatrist, Neuropsychiatric Hospital and \\ WHO Collaborating Centre for Research \& Training in Mental Health, PMB 2002, \\ Abeokuta, Nigeria
}

This century has been characterised by a substantial increase in the number of elderly residents in the community in the technologically advanced countries. One obvious consequence of this has been a pressure on existing mental and socio physical facilities that cater for the elderly. The medico-social factors responsible for this rise in the proportion of the elderly in advanced countries are subtly creeping into many developing countries like Nigeria (Lambo, 1966). It is with this perspective in view, coupled with the dearth of literature on psychogeriatrics in developing countries, that I decided to review the sociodemographic and clinical aspects of elderly (60 years and above) admissions to the Nigerian National Neuropsychiatric Hospital and WHO Collaborating Centre for Research and Training in Mental Health over a five year period (1 January 1982 to 31 December 1986). An attempt will also be made to draw comparisons with my experience of psychogeriatrics in the United Kingdom (I trained in psychiatry at the Royal Edinburgh Hospital in the early 1980s).

\section{The study}

Modern psychiatric practice in Nigeria began at this National Neuropsychiatric Hospital, Abeokuta, in 1954. The hospital has no defined catchment area. It has a total bed complement of about 500 under the aegis of four consultant psychiatrists.

1 January 1982 was selected as the starting point of the review because the patients' clinical records seemed most comprehensive from about this date onwards. All the elderly admissions over the five-year period were identified from the central admissions and discharges register. Relevant information was then extracted from their case notes into a predesigned questionnaire and the ensuing data analysed.

Of the 159 patients admitted over the five-year period, it was possible to retrieve the case notes of $152(96 \%)$. These 152 patients had a total of 169 admissions (3\% of total hospital admissions over the same period).

There were $66(43 \%)$ males and $86(7 \%)$ females. Their ages ranged from 60-85 years (mean and s.d. $=67.1$ years \pm 5.5 ). The single largest group, $72(43 \%)$ was under 65 years. The patients came from six different states in Southern Nigeria and two states in Northern Nigeria, with only about one-third from the state in which the hospital is located.

Eighty-three were married, 35 were widowed, 20 were either separated or divorced and two were never married. Married males significantly outnumbered married females $(P<0.05)$ and widowed females highly significantly outnumbered their male counterparts $(P<0.001)$. The majority $107(70 \%)$ were unskilled workers, mostly peasant farmers or petty traders.

Ninety-eight (58\%) had an associated physical pathology (e.g. fever, anaemia), either at admission or during in-patient stay, while 71 had none. Physical examination was conducted on the patients either at the time of admission or later during the in-patient stay in $65(39 \%)$ while $104(62 \%)$ were never physically examined throughout the period of admission. Of the 65 physically examined, 36 examinations were carried out within 24 hours of admission while the remaining 29 patients had theirs between two and 318 days post admission. Period spent in hospital ranged from $1-1,846$ days (mean and s.d. $=77.1$ days \pm 172.5 )

In the 169 admissions, the family was rated as supportive in $157(93 \%)$ (i.e. contacted patient at least about once every four to six weeks), unsupportive in eight and undetermined in four.

The majority suffered from functional psychoses (schizophrenia-22\%; affective psychoses (mostly depressive) $-22 \%$, paraphrenia - $17 \%$ ). Only about $10 \%$ were diagnosed as suffering from senile dementia while neurosis was non-existent.

One hundred and thirty-one $(78 \%)$ improved by the time they were discharged: 16 did not improve, six were transferred to a General Hospital (mainly because of physical pathology) and $15(9 \%)$ died. The latter comprised seven males and eight females and of these, physical examination was carried out on seven, two of which were done within 24 hours of admission while the remaining five had theirs later.

Of those who improved, the married significantly outnumbered the separated/divorced and the widowed $(P<0.05$ in both instances). Also, those with associated physical pathology significantly outnumbered those without among the ranks of the dead patients $(P<0.05)$. 


\section{Comments}

It is apparent from this review that psychogeriatric practice at the Nigerian National Neuropsychiatric Hospital is not based on a catchment-area approach as is the practice in the UK health service system. Two-thirds of the Nigerian patients were from seven other states within the Federation, all of which had a functioning psychiatric facility during the period of study. The implication of this phenomenon, whereby psychogeriatric patients travel long distances on roads some of which are in a poor state of motorability, gives cause for some disquiet. It may make relatives' contact during periods of admission burdensome, a situation that will not augur well for the patient. Equally, the long distance may handicap home-based assessment of the patient, an approach which is useful in the assessment of the psychogeriatric patient. As ignorance about existing facilities may underly this phenomenon, it is necessary to disseminate information at the grassroots on the location of psychiatric facilities and the benefits of receiving treatment at those facilities that are nearer their domicile.

In this review, all the elderly patients were accompanied to the hospital by either blood relative(s), spouse or an in-law except two patients who came unaccompanied, and one who was accompanied by a neighbour. It is thus obvious that social welfare agencies played virtually no role in the admissions, unlike in the UK where their input into this process may be more important. This may suggest that in Nigeria the extended family system is still actively functional.

In contrast with the Newcastle findings (Kay et al, 1964) which reported that the majority of their elderly patients with psychiatric disorder tended to be either single, separated, divorced or widowed, the majority of these Nigerian patients were still married at the time of their admission. The presence of more widows in the former study may reflect the sociocultural practice of polygamy which is prevalent in Nigeria and in this context, a man will only be labelled as a widower when all his harem are deceased.

It is disquieting that only four out of every ten patients in this study had physical examinations carried out on them during their admission, despite the high prevalence of associated physical pathology present. Of those examined, the examinations tended to be delayed until there was an emergence of associ- ated physical pathology. One obvious difference in psychiatric clinical practice between psychiatrists in the UK and those in developing countries is the heavier patient load on the latter as a result of the lower psychiatrist:patient ratio in the latter situation. A consequence of this is that the psychiatrist in the developing country is sometimes cursory in his interaction with patients and this may account for the large number of cases that were not physically examined in this study. It will be necessary to sound the caveat that psychiatrists in developing countries cannot afford to be cursory in their approach to the elderly who are in fact more vulnerable to relatively minor problems that young adult patients more easily overcome.

The majority of these patients suffered from functional psychoses, an observation in agreement with the Newcastle findings. Neurosis was nonexistent in the present survey. This may be because such disorders are less likely to present to a psychiatric unit in Nigeria because of stigma and the tendency for somatisation which may lead to presentation at general hospitals and/or Trado-spiritual healers.

Finally, it is disturbing to note that these patients in whom associated physical pathology was rife were sometimes admitted into wards manned by nurses with a qualification only in psychiatric nursing. Such nurses will be ill equipped to respond to some of the physical emergencies that the elderly may present on the ward. This may call for the establishment of a separate psychogeriatric unit within the hospital. The majority of the nurses in such a unit should have an additional qualification in general nursing and the nurse to patient ratio should be slightly higher than in a general psychiatric ward to enable staff to cope with the dependencies often presented by the psychogeriatric patient. Such a measure should certainly lead to better care for the elderly psychiatric patient in the Nigerian context.

\section{References}

KaY, D. W. K., Beamish, P. \& Roth, M. (1964). Old age mental disorders in Newcastle upon Tyne. Part II: A study of possible social and medical causes. British Journal of Psychiatry, 110, 668-682.

LAMBO, T. A. (1966) Psychiatric disorders in the aged: Epidemiology and preventive measures. West African Medical Journal, June 1966, 121-124. 